\title{
Inhalt / Sommaire
}

Jakob Büchi, Die wiederaufgefundene «Pharmacia Contracta» des Johann Jakob Scheuchzer (1672-1733)

Adolf Faller, Die Präparation der weißen Substanz des Gehirns bei Stensen, Willis und Vieussens

Luigi Belloni, Il Morgagni tra il Malpighi e il Cotugno

Zdenko Levental, Medizinisches in den Schriften Edward Brownes (1642-1708)

Friedrich Rintelen, Zur Persönlichkeit Karl Gustav Jungs

Walter Nußbaum, Die Grippe-Epidemie 1918/1919 in der schweizerischen Armee

Karl von Meyenn, Die Rezeption der Wellenmechanik und Schrödingers Reise nach Amerika im Winter 1926/27

Peter G. Waser, Walter Rudolf Hess. Aus seinem Leben und seiner Tätigkeit an der Medizinischen Fakultät der Universität Zürich

Delfo Tesi, Augustin-Pyramus de Candolle: Essai d'élaboration d'une taxonomie théorique au $\mathrm{XIX}^{\mathrm{e}}$ siècle

\section{Buchbesprechungen}

Axel Hinrich Murken, Die bauliche Entwicklung des deutschen

Juan L. Carrillo und Luis Gracia Ballester, Enferemedad y Sociedad en la Malaga de los Siglos XVIII + XIX. La Fiebre Amarilla (1741-1821)

(Erwin H. Ackerknecht)

Florian Tennstedt, Sozialgeschichte der Sozialpolitik in Deutschland. Vom 18. Jahrhundert bis zum Ersten Weltkrieg (Erwin H. Ackerknecht)

Heinrich Schipperges, Weltbild und Wissenschaft. Eröffnungsreden zu den Naturforscherversammlungen 1822 bis 1972 (Carl Haffter)

Renato Giuseppe Mazzolini, The Iris in Eighteenth-century Physiology (Urs Boschung)

Renato G. Mazzolini und Guiseppe Ongaro (Hrsg.), Carteggio con Leopoldo Marc'Antonio Caldani (1758-1794) - Peter K. Knoefel, Felice Fontana 1730-1805, An Annotated Bibliography (Urs Boschung)

József Antall, Bilder aus der Geschichte der europäischen Heilkunde und Pharmazie (Urs Boschung)

L. Pecorella Vergnano, L.Belloni, M.L.Turchetti Grossi, Libri e Manoscritti di Haller (Urs Boschung)

Hanspeter Ruesch, Lebensverhältnisse in einem frühen schweizerischen Industriegebiet (Guido Sigron)

Nachrufe

Maria Luisa Righine Bonelli (Maria Celeste Cantù)

Corrigenda 